\title{
Predominance of multi-drug-resistant LAM and Beijing family strains among Mycobacterium tuberculosis isolates recovered from prison inmates in Tula Region, Russia

\author{
Anna Ignatova, ${ }^{1}$ Svetlana Dubiley, ${ }^{1}$ Valentina Stepanshina ${ }^{2}$ \\ and Igor Shemyakin ${ }^{2}$ \\ ${ }^{1}$ Shemyakin-Ovchinikov Institute of Bioorganic Chemistry, Moscow, Russia \\ ${ }^{2}$ State Research Center for Applied Microbiology, Obolensk, Moscow region, Russia
} \\ Correspondence \\ Svetlana Dubiley \\ lana@ibch.ru \\ Received 12 February 2006 \\ Accepted 14 June 2006
}

\section{INTRODUCTION}

Tuberculosis (TB) is one of the most prevalent infections among adults worldwide. An epidemic has been growing in past decades and the spread of multi-drug-resistant (MDR) forms (i.e. resistant to at least rifampicin and isoniazid) of the disease has been remarkable. Russia is recognized by the World Health Organization as a country with a high incidence of TB. Insufficient funding of the healthcare system, lack of continuous population surveillance and delayed diagnostics, together with the AIDS epidemic, have led to the spread of TB. Each year, more than 20000 people die from TB in Russia, corresponding to about $80 \%$ of infectious disease-related mortality (Shilova, 2003).

TB remains a social disease. Prisons serve as TB reservoirs and thus have a significant impact on the incidence of TB in the civilian population. Several outbreaks of TB have been documented worldwide in communities and hospitals where the source was probably a prison inmate (Portugal

\footnotetext{
Abbreviations: ASD-PCR, allele-specific depletory PCR; ETR, exact tandem repeat; FP, fingerprint pattern; MDR, multi-drug resistant; PGG, principal genetic group; RRDR, rifampicin resistance-determining region; ST, shared type; TB, tuberculosis.

Tables showing sequences of primers used, drug resistance profile and genotypic characteristics of the $M$. tuberculosis isolates and prevalence of drug resistance according to spoligofamily and case category and a figure showing identification of principal genetic groups using ASDPCR are available as supplementary material in JMM Online.
}

et al., 1999; Ikeda et al., 1995). Overcrowding, poor ventilation systems, malnourishment, late case finding and inadequate treatment are common in Russian penitentiaries (Bobrik et al., 2005). As a result, the incidence of TB cases in penitentiaries is substantially higher than that for the entire country (Kononets et al., 2004).

Drug-resistant TB is an emerging global problem and Russia is not an exception. Inadequate treatment is believed to be the primary cause of acquired drug resistance. Many MDR $\mathrm{TB}$ cases are identified only when patients fail to respond to conventional therapy, resulting in increased transmission of MDR strains. The spread of MDR TB in prison settings is of major concern. In 2003, $15 \%$ of newly registered cases in Russian prisons were MDR (Kononets et al., 2004).

Drug-resistant TB poses a serious burden on the economy. Treatment of MDR TB requires up to 2 years of therapy, involving up to 100 -fold more expensive treatment regimes compared with non-resistant TB (Chaulk \& Kazandjian, 1998). Early detection of MDR TB cases is a high-priority component of TB control programmes. Phenotypic drug susceptibility testing can take 4-8 weeks (Hacek, 1992; Kent \& Kubica, 1985). The need for fast, inexpensive and userfriendly methods for identification of MDR TB is obvious. The application of molecular tools has the potential to decrease the time necessary for detection of drug resistance of Mycobacterium tuberculosis to a few days or even less, thus a patient's treatment regimen could be adjusted more rapidly and further transmission could be prevented. 
We report here the genotypic characteristics and drug susceptibility profiles of clinical isolates of M. tuberculosis from a prison hospital in the Tula region (central Russia) during 2001 and 2002. In addition, we assessed an in-house PCR-based assay (Dubiley et al., 2005) for determining the mutations associated with resistance to rifampicin and isoniazid that are predominant in central Russia.

\section{METHODS}

Patients. The study included 87 culture-positive patients with pulmonary TB who were diagnosed and treated in the Ozerki prison hospital (Tula region) between June 2001 and June 2002. A total of 103 patients with pulmonary $\mathrm{TB}$ were admitted to the hospital during this period. Specimens were collected once from each of the 103 patients and forwarded to the laboratory after removal of patient identifiers. Sixteen of the collected specimens were considered to be contaminated and were excluded from the analysis.

Bacterial strains, drug susceptibility testing and molecular characteristics of rifampicin and isoniazid resistance. $M$. tuberculosis isolates were cultivated on Lowenstein-Jensen medium. M. tuberculosis was confirmed using a combination of growth, macroscopic and microscopic appearance and biochemical characterization. The M. tuberculosis H37Rv strain was used as a reference for all microbiological and molecular tests.

Drug susceptibility testing was performed by using the absolute concentration assay as described by Kent \& Kubica, 1985. Drug concentrations used were as follows: $1 \mu \mathrm{g}$ isoniazid $\mathrm{ml}^{-1}, 5 \mu \mathrm{g}$ streptomycin $\mathrm{ml}^{-1}, 30 \mu \mathrm{g}$ kanamycin $\mathrm{ml}^{-1}$ and $20 \mu \mathrm{g}$ rifampicin $\mathrm{ml}^{-1}$.

The rifampicin resistance-determining region (RRDR) of $r p o B$ and codons $300-500$ of kat $G$ were analysed for the presence of mutations by sequencing PCR-amplified fragments of the genes. Reactions were carried out using a CycleReader DNA Sequencing kit (Fermentas), according to the manufacturer's protocol. rSfor and rSrev primers were used for sequencing the segment of $r p o B$, and kSfor and $\mathrm{kSrev}$ for the $k a t G$ fragment (primer sequences are available in Supplementary Table S1 in JMM Online).

The allele-specific depletory PCR assay (ASD-PCR) targeting codons 516, 526 and 531 of $r p o B$ and codon 315 of kat $G$ was performed as described previously (Dubiley et al., 2005).

Molecular epidemiology. IS6110-RFLP was performed according to the standard protocol as described by van Embden et al. (1993). Fragment lengths were determined against an external molecular size marker ranging from $0 \cdot 7$ to $15 \cdot 1 \mathrm{~kb}$. Fingerprints were scanned and analysed using GelCompar II software (version 2.5; Applied Maths). Patterns were compared by using the unweighted pair group method of arithmetic means ( $1 \%$ band position tolerance), with Jaccard's similarity index.

Spoligotyping was performed as described elsewhere (Kamerbeek et al., 1997). The number of tandem repeats at each of the exact tandem repeat (ETR) A to E VNTR loci was determined as described by Frothingham \& Meeker-O'Connell (1998). The number of $77 \mathrm{bp}$ repeats only was counted in the ETR-D locus.

An in-house ASD-PCR assay targeting polymorphic nucleotides in codon 95 of gyrA and codon 463 of kat $G$ was used for identification of the principal genetic group (PGG; Sreevatsan et al., 1997). Two complementary PCR reactions differing in allele-specific primer sets were carried out for identification of polymorphisms in these positions. The PCR mixtures contained $0 \cdot 1 \mu \mathrm{M}$ each katG-Rev and gyrA-Rev primers, $0 \cdot 2 \mu \mathrm{M}$ each ISFor and ISRev primers, approximately $10 \mathrm{ng}$ genomic DNA, $40 \mu \mathrm{M}$ each dNTP and $2 \mathrm{U}$ Taq DNA polymerase (Promega) in the buffer recommended by the manufacturer. In addition to those listed above, the first PCR reaction mixture contained $0 \cdot 5 \mu \mathrm{M}$ each R463katG and S95gyrA primers and the complementary one contained the same concentration of each L463katG and T95gyrA allele-specific primers (primer sequences are available in Supplementary Table S1 in JMM Online). Amplification was carried out under the following conditions: initial denaturation at $96^{\circ} \mathrm{C}$ for $5 \mathrm{~min}$, then $30-40$ cycles with denaturation at $95^{\circ} \mathrm{C}$ for $40 \mathrm{~s}$, annealing at $62{ }^{\circ} \mathrm{C}$ for $20 \mathrm{~s}$ and elongation at $72{ }^{\circ} \mathrm{C}$ for $60 \mathrm{~s}$. PCR products were detected by using electrophoresis in ethidium bromide-stained $1 \%$ agarose gels (see Supplementary Fig. S1 in JMM Online).

\section{RESULTS}

\section{Study population}

The 87 patients included in this study were male, aged between 20 and 56 years (mean age, 35.9 years). All were residents of the Tula region and were incarcerated in the prison facility located in this region; their terms of imprisonment ranged from several months to 14 years. Pulmonary consolidation and fibrocavitary forms of TB were observed in $76(87 \cdot 4 \%)$ and $8(9 \cdot 2 \%)$ cases, respectively. None of the patients were HIV-seropositive. Patients were divided into two groups [as recommended by the World Health Organization (1993)]. The first group comprised newly diagnosed sputum smear-positive patients who had not received anti-TB medication previously or had been treated for less than a month. The second group comprised smear-positive patients who had previously taken one or more drugs for more than 1 month. Sixtythree patients had been treated previously and 24 were newly diagnosed cases. No substantial difference in terms of imprisonment was found in these groups. The mean age of newly diagnosed patients was similar to that of previously treated patients $(34 \cdot 2$ and $36 \cdot 7$ years, respectively).

\section{Genotypic classification of strains}

Isolates were assigned to one of three principal genetic groups based on the combinations of polymorphisms at kat $G$ codon 463 ( $\mathrm{R}>\mathrm{L}, \mathrm{CGG}>\mathrm{CTG})$ and gyrA codon 95 $(\mathrm{S}>\mathrm{T}, \mathrm{AGC}>\mathrm{ACC})$. Five isolates $(5 \cdot 7 \%)$ were found to belong to PGG 3 , and $44(50 \cdot 6 \%)$ and $38(43 \cdot 7 \%)$ to PGG 2 and 1 , respectively. Detailed genotypic characteristics of each strain are presented in Supplementary Table S2 in JMM Online.

IS6110-RFLP analysis of the 87 isolates revealed 45 distinct IS6110 fingerprint patterns (FPs). The number of IS6110 copies per genome varied from 8 to 20 , with a mean of 12.56 insertions. Twelve clusters composed of $100 \%$ identical IS6110 patterns were identified. VNTR- and spoligotyping results are summarized in Table 1. Spoligotyping of 87 isolates produced 13 distinct profiles. Spoligopatterns were compared with those published in the International Spoligotyping Database of the Institute Pasteur de Guadeloupe (Brudey et al., 2006). Two isolates $(2 \cdot 3 \%)$ 
Table 1. Genotypic characteristics of M. tuberculosis isolates

PGG, Principal genetic group; ST, shared type.

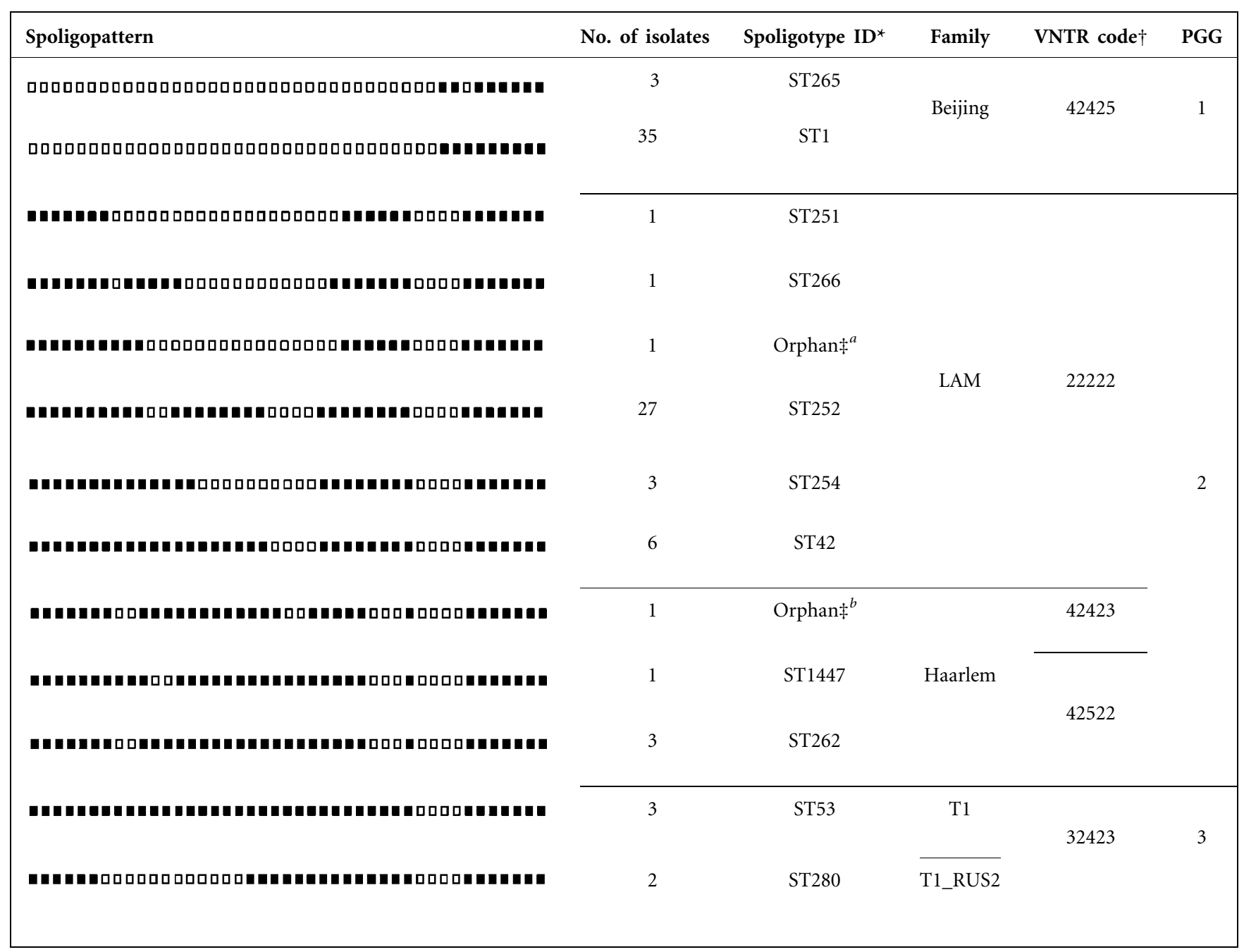

${ }^{*}$ Identified according to the International Spoligotyping Database of the Institute Pasteur de Guadeloupe.

$\dagger$ Number of tandem repeats at each of ETR A to E VNTR loci.

$\ddagger$ Spoligotype not present in the International Spoligotyping Database: $a$, isolate no. 741; $b$, isolate no. 755 .

had unique spoligopatterns not found in the database, whereas $85(97 \cdot 7 \%)$ patterns were of the shared type (ST). Two major spoligotypes, represented by 35 and 27 isolates, were ST1 (Beijing) and ST252 (LAM09), respectively.

Thirty-eight isolates $(43 \cdot 7 \%)$ had spoligopatterns characteristic of the Beijing family (ST1 and 265). Two families, T1_RUS2 and T1, were represented by two $(2 \cdot 3 \%)$ and three isolates $(3 \cdot 4 \%)$, respectively. Supplementary Table S2 in JMM Online shows that $39(44 \cdot 8 \%)$ isolates comprised a clearly distinguishable group according to IS6110-RLFP patterns. They shared identical VNTR profiles (22222). All these isolates belonged to PGG 2 (Table 1). This allowed us to recognize ST251, ST266, ST254, ST252, ST42 and isolate no. 741 as members of the LAM family. Similarly, a group of five isolates (ST262, ST1447 and isolate no. 755) was attributed to the Haarlem family.

\section{Phenotypic and genotypic characterization of drug resistance}

Analysis of drug resistance in relation to case category revealed that the highest rate of resistance was to streptomycin, followed by isoniazid and rifampicin for both new and previously treated cases. Results of drug resistance testing are summarized in Table 2 (for detailed data on each strain see Supplementary Table S2 in JMM Online). Overall, the rate of resistance to individual drugs was similar or slightly lower than that reported for Serpukhov prison (Shemyakin et al., 2004). Two isolates $(2 \cdot 3 \%)$ were resistant only to streptomycin and two isolates $(2 \cdot 3 \%)$ were rifampicin-monoresistant. No monoresistance to isoniazid or kanamycin was observed. Forty-nine isolates $(56 \cdot 3 \%)$ were resistant to the four drugs tested. All MDR isolates were resistant to streptomycin. The MDR rate was 
Table 2. Prevalence of drug resistance in new and previously treated cases

INH, Isoniazid; KAN, kanamycin; MDR, multi-drug resistant; RIF, rifampicin; STR, streptomycin.

\begin{tabular}{|lccc|}
\hline Drug resistance & New cases $(\boldsymbol{n}=\mathbf{2 4 )}$ & Previously treated cases $(\boldsymbol{n}=\mathbf{6 3})$ & Total $(\boldsymbol{n}=\mathbf{8 7})$ \\
\hline Susceptible & $6(25 \cdot 0 \%)$ & $2(3 \cdot 2 \%)$ & $8(9 \cdot 2 \%)$ \\
STR & $17(70 \cdot 8 \%)$ & $60(95 \cdot 2 \%)$ & $77(88 \cdot 5 \%)$ \\
INH & $12(50 \cdot 0 \%)$ & $55(87 \cdot 3 \%)$ & $67(77 \cdot 0 \%)$ \\
KAN & $9(37 \cdot 5 \%)$ & $49(77 \cdot 8 \%)$ & $58(66 \cdot 7 \%)$ \\
RIF & $10(41 \cdot 7 \%)$ & $56(88 \cdot 9 \%)$ & $66(75 \cdot 9 \%)$ \\
MDR & $9(37 \cdot 5 \%)$ & $53(84 \cdot 1 \%)$ & $62(71 \cdot 2 \%)$ \\
\hline
\end{tabular}

$37 \cdot 5 \%$ among new cases, which was similar to that reported in Archangelsk (34\%) and Samara (37.3\%). The MDR rate among previously treated cases was higher $(84 \cdot 1 \%$ in Ozerki versus 55\% in Archangelsk and 53.6\% in Samara) reflecting an even worse situation with TB treatment in the Ozerki prison compared with the other cited penitentiaries in Russia.

PCR and sequencing techniques were used to determine the mutations conferring rifampicin and isoniazid-resistance in the isolates studied. All isolates were subjected to an ASDPCR assay targeting codons 516, 526 and 531 of rpoB and codon 315 of kat $G$ and sequencing of the RRDR of $r p o B$ and a fragment of katG. The ASD-PCR and sequencing data showed that all isoniazid-resistant strains (67/67) carried the $\mathrm{S}>\mathrm{T}$ substitution in codon 315 of katG. No other mutations were found in the kat $G$ segment analysed. Results of the mutation analysis in $r p o B$ are summarized in Table 3. Most rifampicin-resistant isolates in this study $(55 / 66,83 \cdot 3 \%)$ carried the D516V or S531L allele of rpoB. No mutations in RRDR were detected in five rifampicin-resistant isolates. Two of these isolates were rifampicin-monoresistant, one was resistant to both rifampicin and streptomycin, and two were resistant to three drugs, rifampicin, streptomycin and isoniazid. The results obtained by ASD-PCR were in complete agreement with the sequencing data.

\section{Molecular epidemiology}

Fifty-four isolates $(62 \cdot 1 \%)$ were members of the 12 clusters identified. The number of isolates per cluster varied from 2 to 17 , with a mean of $4 \cdot 5$. Seven clusters consisted of 2 isolates, 3 clusters consisted of 6 or more isolates. No significant difference in the ratio of multi-drug resistance or resistance to individual drugs was detected between clustered and unique isolates. In some cases, members of the same cluster showed different drug susceptibility profiles. For example, the major Beijing cluster, FP25, comprised two fully susceptible, one rifampicin-monoresistant and five MDR strains. Members of the second major Beijing cluster, FP42, shared similar drug susceptibility profiles but had different mutations in the $r p o B$ gene. These data indicate that this particular strain acquired rifampicin resistance after dissemination. The number of new cases was slightly higher among clustered isolates (29.6 versus $24 \cdot 3 \%)$. Two of eight (25\%) unique new cases were MDR.

In some cases, ignoring close similarities between IS6110 patterns may result in underestimation of the epidemiological links between patients (Yeh et al., 1998). It has been shown in a number of studies (Niemann et al., 2000; Yeh et al., 1998; Warren et al., 2002) that up to four IS6110 banding pattern changes (appearance, disappearance or shift of a band) may occur during recent transmission or persistent disease. It has been demonstrated that about $20 \%$ of transmission events produced variant strains (van der Spuy et al., 2003). About $31 \%$ (27/87) of isolates recovered in the prison hospital shared identical spoligotype ST252, attributed to the LAM09 spoligofamily. According to IS6110 typing, two clusters (FP7 and FP8) and five unique FPs are contained in this group of isolates. The overall similarity in this group was $85 \%$. The genetic distance between the isolates [number of mismatched bands in IS6110 patterns

Table 3. Mutations in rpoB of rifampicin-resistant isolates of $M$. tuberculosis, according to spoligofamily

\begin{tabular}{|lcccc|}
\hline Mutation & Beijing $(\boldsymbol{n}=\mathbf{3 1})$ & LAM $(\boldsymbol{n}=\mathbf{3 4})$ & Other $(\boldsymbol{n}=\mathbf{1})$ & Total $(\boldsymbol{n}=\mathbf{6 6})$ \\
\hline L511P & $1(3 \cdot 2 \%)$ & $0(0 \cdot 0 \%)$ & $0(0 \cdot 0 \%)$ & $1(1 \cdot 5 \%)$ \\
D516V & $0(0 \cdot 0 \%)$ & $27(79 \cdot 4 \%)$ & $0(0 \cdot 0 \%)$ & $27(40 \cdot 9 \%)$ \\
H526Y & $1(3 \cdot 2 \%)$ & $0(0 \cdot 0 \%)$ & $0(0 \cdot 0 \%)$ & $1(1.5 \%)$ \\
H526D & $2(6 \cdot 5 \%)$ & $2(5 \cdot 9 \%)$ & $0(0 \cdot 0 \%)$ & $4(6 \cdot 1 \%)$ \\
S531L & $24(77 \cdot 4 \%)$ & $4(11 \cdot 8 \%)$ & $0(0 \cdot 0 \%)$ & $28(42 \cdot 4 \%)$ \\
Not identified & $3(9 \cdot 7 \%)$ & $1(2 \cdot 9 \%)$ & $1(100 \cdot 0 \%)$ & $5(7 \cdot 6 \%)$ \\
\hline
\end{tabular}

${ }^{\star}$ No mutations in the RRDR were detected. 
(van der Spuy et al., 2003)] did not exceed three, thus allowing consideration of this group as a supercluster. These isolates shared drug-resistance patterns. All of them were resistant to streptomycin, rifampicin, isoniazid and kanamycin and all of them carried the mutation D516V in $r p o B$ and $\mathrm{S} 315 \mathrm{~T}$ substitution in katG. Retrospective analysis of the isolates recovered in the Serpukhov prison (Shemyakin et al., $2004)$ showed that $11 \cdot 5 \%(13 / 113)$ of them belonged to this supercluster and had identical drug-resistance profiles. The two prisons are $120 \mathrm{~km}$ apart. These data support the assumption that a particular parental strain had been circulating in the environment for a prolonged time and had been split into a number of variant strains. The data above suggest that this strain may present a higher risk of transmission with respect to other strains found in the study.

\section{DISCUSSION}

Difficulties in establishing transmission links among prisoners are well known (Jones et al., 1999). Only limited clinical information on patients was available in this study and patients' contacts were not identified. However, the high proportion of clustered isolates and the spread of drug resistance in new cases suggest active transmission among the prisoners.

Two families of M. tuberculosis strains were predominant among the isolates analysed. The Beijing/W family of strains is known for its frequent association with multi-drug resistance and high emergence worldwide (Glynn et al., 2002). Several outbreaks associated with this family have been reported (Bifani et al., 1996; Milan et al., 2004). The prevalence of Beijing isolates in Russia, both in prisons and in the community, and the high rate of multi-drug resistance typical for this group have been recognized in a number of studies (Toungoussova et al., 2003; Ruddy et al., 2005; Niemann et al., 2000; Shemyakin et al., 2004). Our results were consistent with this observation $-43 \cdot 7 \%(38 / 87)$ of isolates were attributed to the Beijing family and $76 \cdot 3 \%$ (29/ 38) of them were MDR (Supplementary Table S3 in JMM Online).

Whereas a considerable amount of information is available on the Beijing/W family, the same is not true for other families. Limited data are available on epidemiological and molecular characteristics of the LAM family. Our results together with the data reported previously show that this family is widely spread in central Russia (Shemyakin et al., $2004)$. In our study, $44 \cdot 8 \%(39 / 87)$ of isolates were members of the LAM family. Drug susceptibility testing has revealed a high rate of MDR isolates in this group (33/39, $84 \cdot 6 \%)$. Fifty seven per cent of the new cases (4/7) and $90.6 \%(29 / 32)$ of previously treated cases were MDR. Overall, $100 \%$ of MDR isolates among both previously treated and new cases belonged to the Beijing or LAM families (see Supplementary Table S3 in JMM Online).

The high rate of drug-resistant TB in the prison setting is a major concern. Treatment of MDR TB is complex. Patients with MDR TB remain infectious for a longer time, increasing the probability of spread of infection. Early identification and isolation of MDR TB cases and switching to second-line drug therapy is an important part of a TB control programme. Molecular methods may speed up the diagnosis of drug resistance. The main limitation of any genotypic technique that aims to detect drug resistance is that it can detect only those mutations that are screened for. However, the accurate choice of target mutations enables detection of the majority of resistance. As was shown in a 5 year community-based study of TB in South Africa, more than $90 \%$ of resistance to rifampicin, streptomycin and ethambutol and $75 \%$ of resistance to isoniazid could be detected by assaying six mutations (Van Rie et al., 2001).

An unusually high proportion of the S315T mutation in $k a t G$ is characteristic of isoniazid-resistant strains in Russia (Nikolayevsky et al., 2004). According to previously reported data (Generozov et al., 2000), mutations in codons 516, 526 and 531 of $r p o B$ are responsible for $86-100 \%$ of rifampicin-resistant TB cases in Russia and are among the most frequently reported in other regions with high rates of MDR TB (Ramaswamy \& Musser, 1998). These mutations were targeted by ASD-PCR. Using this technique, we accurately identified isoniazid-resistant and susceptible isolates (87/87). Rifampicin susceptibility of $81(93 \cdot 1 \%)$ and $82(94.3 \%)$ of 87 isolates was identified correctly by using the ASD-PCR assay and by sequencing, respectively. In comparison with sequencing, only one isolate was not recognized as rifampicin resistant as it contained the substitution in codon 511 of $r p o B$ that was not targeted by the ASD-PCR assay. These results demonstrate that the mutations targeted by ASD-PCR were relevant in this setting. More efforts should be made to prevent the spread of MDR TB and early case finding is a crucial part of the prevention strategy. The introduction of molecular methods of MDR TB diagnostics would improve disease management and decrease the transmission rate.

The Russian Federation has one of the highest incarceration rates in the world. In 2002, there were 640 inmates per 100000 population (Bobrik et al., 2005). Thus, insufficient TB control measures in prison settings have an substantial impact on communities outside prisons, especially in Russia. Our results showed that, in contrast to other geographical regions of Russia, together with Beijing strains, LAM strains were major contributors to the TB epidemiological picture and accounted for most of the drug-resistant TB in the population studied.

\section{ACKNOWLEDGEMENTS}

We thank Dr V. I. Kosarev and Dr I. N. Savel'eva from the Tuberculosis Hospital (Ozerki, Russia) for providing the M. tuberculosis isolates and the patient characteristics required for the study. We thank Dr Thomas M. Shinnick for providing the molecular size marker. We are grateful to Dr Rachel Albalak from CDC, Atlanta, USA for critical reading of the manuscript. This study was supported by the International Science and 
Technology Centre and the Biotechnology Engagement Program of the US Department of Health and Human Services under grant ISTC2628/ BTEP63.

\section{REFERENCES}

Bifani, P. J., Plikaytis, B. B., Kapur, V. \& 10 other authors (1996). Origin and interstate spread of a New York City multidrug-resistant Mycobacterium tuberculosis clone family. JAMA (J Am Med Assoc) 275, 452-457.

Bobrik, A., Danishevski, K., Eroshina, K. \& McKee, M. (2005). Prison health in Russia: the larger picture. J Public Health Policy 26, 30-59.

Brudey, K., Driscoll, J. R., Rigouts, L. \& 63 other authors (2006). Mycobacterium tuberculosis complex genetic diversity: mining the fourth international spoligotyping database (SpolDB4) for classification, population genetics and epidemiology. BMC Microbiol 6, 23.

Chaulk, C. P. \& Kazandjian, V. A. (1998). Directly observed therapy for treatment completion of pulmonary tuberculosis: Consensus Statement of the Public Health Tuberculosis Guidelines Panel. JAMA (J Am Med Assoc) 279, 943-948.

Dubiley, S., Mayorova, A., Ignatova, A., Kirillov, E., Stepanshina, V., Kolesnikov, A. \& Shemyakin, I. (2005). New PCR-based assay for detection of common mutations associated with rifampin and isoniazid resistance in Mycobacterium tuberculosis. Clin Chem 51, 447-450.

Frothingham, R. \& Meeker-O'Connell, W. A. (1998). Genetic diversity in the Mycobacterium tuberculosis complex based on variable numbers of tandem DNA repeats. Microbiology 144, 1189-1196.

Generozov, E. V., Akopian, T. A., Govorun, V. M., Chernousova, L. N., Larionova, E. E., Savinkova, S. N., Smirnova, T. G., Golyshevskaya, V. I. \& Khomenko, A. G. (2000). Molecular characteristics of multiresistant clinical strains of Mycobacterium tuberculosis isolated in Russia. Mol Gen Mikrobiol Virusol part 1, 11-17 (in Russian).

Glynn, J. R., Whiteley, J., Bifani, P. J., Kremer, K. \& van Soolingen, D. (2002). Worldwide occurrence of Beijing/W strains of Mycobacterium tuberculosis: a systematic review. Emerg Infect Dis 8, 843-849.

Hacek, D. (1992). Modified proportion agar dilution test for slowly growing mycobacteria. In Clinical Microbiology Procedures Handbook, vol. 1, pp. 5.13.1-5.13.15. Edited by H. Isenberg. Washington, DC: American Society for Microbiology.

Ikeda, R. M., Birkhead, G. S., DiFerdinando, G. T., Jr, Bornstein, D. L., Dooley, S. W., Kubica, G. P. \& Morse, D. L. (1995). Nosocomial tuberculosis: an outbreak of a strain resistant to seven drugs. Infect Control Hosp Epidemiol 16, 152-159.

Jones, T. F., Craig, A. S., Valway, S. E., Woodley, C. L. \& Schaffner, W. (1999). Transmission of tuberculosis in a jail. Ann Intern Med 131, 557-563.

Kamerbeek, J., Schouls, L., Kolk, A. \& 8 other authors (1997). Simultaneous detection and strain differentiation of Mycobacterium tuberculosis for diagnosis and epidemiology. J Clin Microbiol 35, 907-914.

Kent, P. T. \& Kubica, G. P. (1985). Public Health Mycobacteriology. A Guide for the Level III Laboratory. Atlanta: Centers for Disease Control and Prevention.

Kononets, A. S., Sidorova, S. V. \& Safonova, S. G. (2004). Organization of antituberculous care in penitentiary facilities. Probl Tuberk Bolezn Legk 11, 18-20 (in Russian).

Milan, S. J., Hauge, K. A., Kurepina, N. E. \& 7 other authors (2004). Expanded geographical distribution of the $\mathrm{N}$ family of Mycobacterium tuberculosis strains within the United States. J Clin Microbiol 42, 1064-1068.
Niemann, S., Rüsch-Gerdes, S., Richter, E., Thielen, H., HeykesUden, H. \& Diel, R. (2000). Stability of IS6110 restriction fragment length polymorphism patterns of Mycobacterium tuberculosis strains in actual chains of transmission. J Clin Microbiol 38, 2563-2567.

Nikolayevsky, V., Brown, T., Balabanova, Y., Ruddy, M., Fedorin, I. \& Drobniewski, F. (2004). Detection of mutations associated with isoniazid and rifampin resistance in Mycobacterium tuberculosis isolates from Samara Region, Russian Federation. J Clin Microbiol 42, 4498-4502.

Portugal, I., Covas, M. J., Brum, L., Viveiros, M., Ferrinho, P., MonizPereira, J. \& David, H. (1999). Outbreak of multiple drug-resistant tuberculosis in Lisbon: detection by restriction fragment length polymorphism analysis. Int J Tuberc Lung Dis 3, 207-213.

Ramaswamy, S. \& Musser, J. M. (1998). Molecular genetic basis of antimicrobial agent resistance in Mycobacterium tuberculosis: 1998 update. Tuber Lung Dis 79, 3-29.

Ruddy, M., Balabanova, Y., Graham, C. \& 12 other authors (2005). Rates of drug resistance and risk factor analysis in civilian and prison patients with tuberculosis in Samara Region, Russia. Thorax 60, 130-135.

Shemyakin, I. G., Stepanshina, V. N., Ivanov, I. Y., Lipin, M. Y., Anisimova, V. A., Onasenko, A. G., Korobova, O. V. \& Shinnick, T. M. (2004). Characterization of drug-resistant isolates of Mycobacterium tuberculosis derived from Russian inmates. Int J Tuberc Lung Dis 8, 1194-1203.

Shilova, M. (2003). Tuberculosis in various Federal Districts of Russia. In Abstracts of the 7th Russian Congress of Phthisiatrists, Tuberculosis Today, abstract 31. Moscow: BINOM.

Sreevatsan, S., Pan, X., Stockbauer, K. E., Connell, N. D., Kreiswirth, B. N., Whittam, T. S. \& Musser, J. M. (1997). Restricted structural gene polymorphism in the Mycobacterium tuberculosis complex indicates evolutionarily recent global dissemination. Proc Natl Acad Sci U S A 94, 9869-9874.

Toungoussova, O. S., Mariandyshev, A., Bjune, G., Sandven, P. \& Caugant, D. A. (2003). Molecular epidemiology and drug resistance of Mycobacterium tuberculosis isolates in the Archangel prison in Russia: predominance of the W-Beijing clone family. Clin Infect Dis 37, 665-672.

van der Spuy, G. D., Warren, R. M., Richardson, M., Beyers, N., Behr, M. A. \& van Helden, P. D. (2003). Use of genetic distance as a measure of ongoing transmission of Mycobacterium tuberculosis. J Clin Microbiol 41, 5640-5644.

van Embden, J. D. A., Cave, M. D., Crawford, J. T. \& 8 other authors (1993). Strain identification of Mycobacterium tuberculosis by DNA fingerprinting: recommendations for a standardized methodology. J Clin Microbiol 31, 406-409.

Van Rie, A., Warren, R., Mshanga, I. \& 9 other authors (2001). Analysis for a limited number of gene codons can predict drug resistance of Mycobacterium tuberculosis in a high-incidence community. J Clin Microbiol 39, 636-641.

Warren, R. M., van der Spuy, G. D., Richardson, M., Beyers, N., Booysen, C., Behr, M. A. \& van Helden, P. D. (2002). Evolution of the IS6110-based restriction fragment length polymorphism pattern during the transmission of Mycobacterium tuberculosis. J Clin Microbiol 40, 1277-1282.

World Health Organization (1993). Treatment of Tuberculosis. Guidelines for National Programs. Geneva: World Health Organization.

Yeh, R. W., Ponce de Leon, A., Agasino, C. B., Hahn, J. A., Daley, C. L., Hopewell, P. C. \& Small, P. M. (1998). Stability of Mycobacterium tuberculosis DNA genotypes. J Infect Dis 177, 1107-1111. 